\title{
Ultrashort soliton switching based on coherent energy hiding
}

\author{
M. Romagnoli, S. Wabnitz, and L. Zoccolotti \\ Fondazione Ugo Bordoni, Via B. Castiglione 59, 00142 Rome, Italy
}

Received April 24, 1991

\begin{abstract}
Coherent coupling between light and atoms may be exploited for conceiving a novel class of all-optical signalprocessing devices without a direct counterpart in the continuous-wave regime. We show that the self-switching of ultrashort soliton pulses on resonance with a transition of doping centers in a slab waveguide directional coupler is based on nonlinear group-velocity (instead of the usual phase-velocity) changes.
\end{abstract}

The nonlinear response of a material to a propagating field permits the control of light by light, which may permit ultrafast all-optical signal processing. For example, Fig. 1(a) shows the intensity-induced routing of two $N=1$ solitons of the nonlinear Schrödinger equation by means of a Kerr nonlinear directional coupler ${ }^{1,2}$ (NLDC). Pulses with relatively low intensities emerge from guide 2. By an increase in the input soliton intensity, the nonlinear refractive-index mismatches the coupler, and coupling is suppressed. In this Letter we demonstrate that an opposite switching behavior may occur in the coherent regime, i.e., whenever the input pulse width is shorter than the decay times of a two-level system (TLS) nonlinearity. ${ }^{3}$ On TLS resonance, the nonlinear change of refractive index is zero, therefore no nonlinear switching is possible for continuous waves. On the other hand, if the input pulse is a $2 \pi$ soliton of self-induced transparency ${ }^{4}$ (SIT), then linear coupling may be suppressed by increasing the input time width of the constant area pulses above a certain threshold value [see Fig. 1(b)].

The possibility of switching SIT solitons of fixed time width in a TLS-doped NLDC by means of controlling the input pulse area was suggested in Ref. 3 . Here we elucidate the physical principle of SIT soliton dynamics in the NLDC and propose that the switching may be controlled by changing the input pulse width (or velocity). Moreover, in Ref. 3 an approximate coupled-mode formalism (i.e., valid only in the ideal case of a flat mode and doping profile in the guiding region, and no field in the cladding ${ }^{5,6}$ ) was used. Since coherent pulse propagation is sensitive to transverse effects, ${ }^{4,7,8}$ we consider here realistic one-dimensional slab waveguides and directly solve the wave equation in one spatial transverse, temporal, and longitudinal coordinate.

The equations for the slowly varying envelopes of the field $E(x, z, t)$ and polarization $P(x, z, t)$ at the resonant frequency $\omega$ read

$$
\begin{aligned}
2 \beta\left(\frac{\partial E}{\partial z}+\beta^{\prime} \frac{\partial E}{\partial t}\right) & =i\left[k_{0}{ }^{2} n(x, z)^{2}-\beta^{2}\right] E \\
& +i \frac{\partial^{2} E}{\partial x^{2}}-\sigma E+\alpha^{\prime}(x) P, \\
\frac{\partial P}{\partial t} & =\frac{\wp}{\hbar} E W, \\
\frac{\partial W}{\partial t} & =-\frac{\wp}{2 \hbar}\left(P E^{*}+E P^{*}\right) .
\end{aligned}
$$

Here $\beta$ is the linear propagation constant of the waveguide mode, $1 / \beta^{\prime}$ is the mode group velocity, $k_{0}=\omega / c, x$ is the transverse coordinate, $n(x, z)$ is the linear index profile of the coupler, $\sigma$ is the linear background absorption, $W$ is the population inversion, $\alpha^{\prime}=\wp \mu_{0} \omega^{2} N(x)$, where $\wp$ is the dipole moment of the transition, and $N(x)$ is the doping profile across the coupler. In Eqs. (1) we have neglected the relaxation times for the population and the polarization, say, $T_{1}$ and $T_{2}$. Equations (1) may be reduced to a dimensionless form by changes in the variables,

$$
\begin{gathered}
\frac{\wp \tau_{0} E}{\hbar} \rightarrow E, \quad\left(t-\frac{z}{\beta^{\prime}}\right) / \tau_{0} \rightarrow t, \\
z k_{0} n_{0}=\frac{2 \pi z}{\lambda} \rightarrow z, \quad x k_{0} n_{0} \rightarrow x,
\end{gathered}
$$

where $\tau_{0}$ is an arbitrary time width, subject to $\tau_{0} \ll T_{1}, T_{2}$. Equations (1) reduce to

$$
\begin{aligned}
& \frac{\partial E}{\partial z}=\frac{i}{2 \tilde{\beta}}\left[\left(\tilde{n}^{2}-\tilde{\beta}^{2}\right) E+\frac{\partial^{2} E}{\partial x^{2}}\right]-\tilde{\sigma} E+\alpha P, \\
& \frac{\partial P}{\partial t}=E W, \\
& \frac{\partial W}{\partial t}=-\frac{1}{2}\left(P E^{*}+E P^{*}\right),
\end{aligned}
$$

where we have defined

$$
\begin{aligned}
& \alpha \doteq \frac{\alpha^{\prime} \wp \tau_{0}}{2 k_{0} n_{0} \beta \hbar}, \\
& \tilde{n} \doteq \frac{n}{n_{0}}=(1+\delta n), \\
& \tilde{\beta} \doteq \frac{\beta}{k_{0} n_{0}}, \quad \tilde{\sigma} \doteq \frac{\sigma}{2 \beta k_{0} n_{0}} .
\end{aligned}
$$

We chose the following input conditions for the field and the atoms:

$$
\begin{aligned}
& E(z=0, x, t)=\frac{A}{\tau} \operatorname{sech}(t / \tau) F\left(x-x_{0}\right), \\
& P(z, t=-T)=0, \quad W(z, t=-T)=-1,
\end{aligned}
$$

where $F(x)$ is the transverse profile of the local $\mathrm{TE}_{0}$ mode of an individual guide (with the center at $x=x_{0}$ ) and $T$ is the half-width of the computational 
(a)

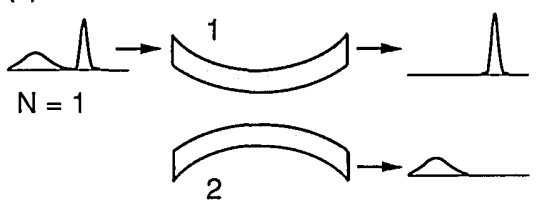

(b)

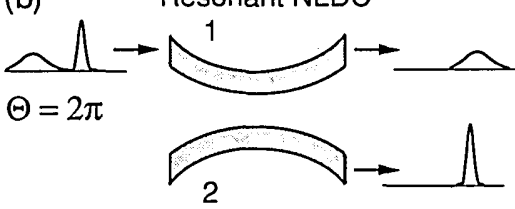

Fig. 1. Switching in a soliton NLDC

(b) two-level system solitons.

(a) Kerr solitons,
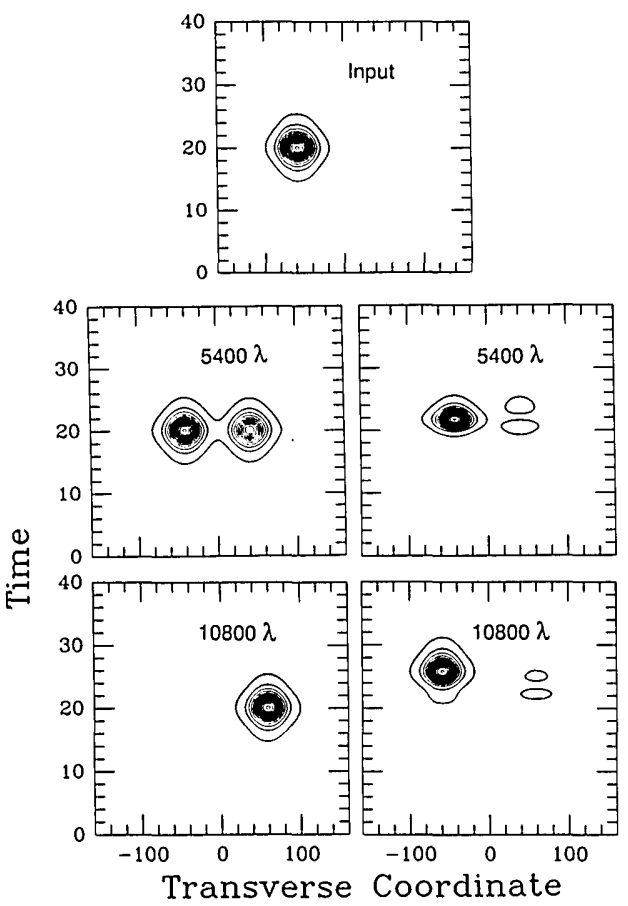

Fig. 2. Input pulse (top) and pulses in the NLDC after one half and one coupling lengths. Left, linear coupler; right, resonant TLS doping, with an input area $\Theta=2 \pi$.

temporal window. The function $F$ was normalized so that the pulse area $\Theta \doteq \int E(x=0) \mathrm{d} t=\pi A$.

In the computations, we have used a grid of 256 samples both in the time and transverse coordinates, a temporal window $2 T=20 \tau$, and a transverse window $2 X=500$. We solved Eqs. (3) by means of a split-step procedure, where the linear diffraction step was advanced by Fourier transformation, whereas the propagation step involving the linear index profile and the nonlinear interaction with the TLS doping was advanced by means of a predictor-corrector method. ${ }^{9}$

We assume here a NLDC with tapering of the coupling strength. That is, we allow for a slow variation with $z$ of the distance $\Delta x=H_{0}\left(1+\gamma z^{2}\right)$ between the two waveguides. A parabolic tapering of the coupler leads to an exponential dependence on $z$ of the linear coupling coefficient as in Ref. 3. We have set for the linear index difference in the guiding region of the identical step index guides $\delta n=$
0.00363 , and the guide width was $h=41.1$, which yields single-mode operation. The minimal centerto-center separation between the guides was $d=$ $H_{0}+h=2 h$, and the bending coefficient was $\gamma=7.5 \times 10^{-10}$. Finally, the peak value of the nonlinearity coefficient was set equal to $\alpha(0)=1.39 \times$ $10^{-4}$, and the total length of the coupler was $L=$ $6.78 \times 10^{4}$. The adiabatic tapering condition, $H_{0} \gamma L \ll 1$, was well satisfied.

In Fig. 2 we show the input pulse and the pulses at one half and one coupling distances (in terms of $\lambda=$ $\left.\lambda_{0} / n\right)$ in the tapered coupler. Here we have taken $\tau=2$ and have assumed that $\sigma=0$. This figure shows a main result of the simulations: an input pulse of area $\Theta=2 \pi$ may travel almost undistorted in the input guide without coupling to the other channel (Fig. 2, right), whereas in the absence of doping the coupling is complete. Note that in each contour plot the intensity is normalized with respect to the peak value. Initially the time width of the soliton is compressed by approximately a factor of 2 , whereas the peak intensity grows larger. On exiting from the coupler, the pulse is slightly absorbed, and its time width recovers the initial value.

Figure 3 shows two examples of output pulse profiles; the input area is $\Theta=4 \pi$ in both cases, but the input time width is different. In Fig. 3(a) the input pulse width is $\tau=4$ so that the initial amplitude is the same as in Fig. 2 (left). By comparing Figs. 2 and $3(\mathrm{a})$, one can see that the bar state for the soli-
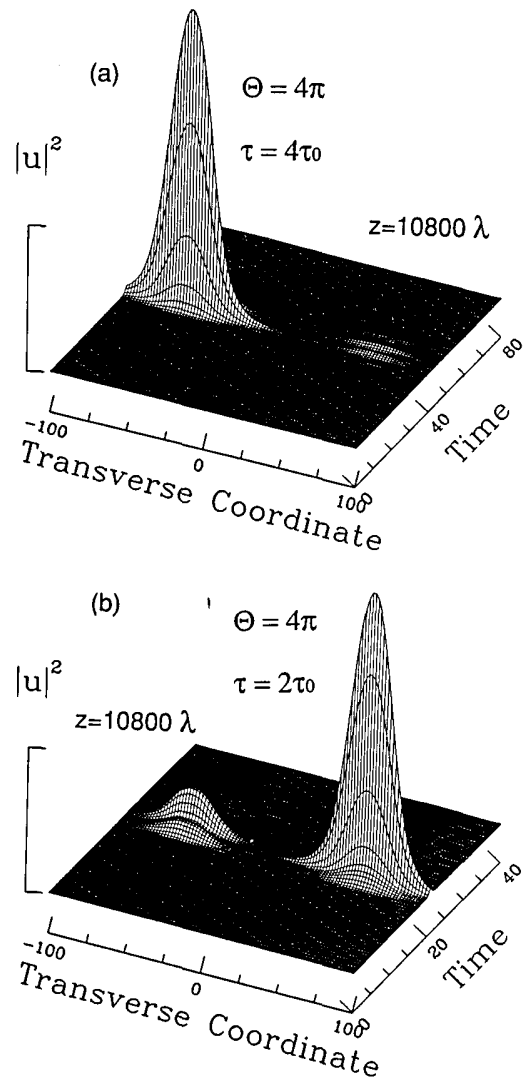

Fig. 3. Switching of the output pulse from the bar to the cross state by halving the input time width of the input pulse (with a constant area $\Theta=4 \pi$ ) for (a) $\tau=4 \tau_{0}$ and (b) $\tau=2 \tau_{0}$. 
ton is stable with respect to an increase of the pulse width. On the other hand, Fig. 3(b) shows the important property that, if the input area is maintained constant but the pulse width is reduced, switching of the soliton from the bar to the cross state may be obtained [see Fig. 1(b)]. In Fig. 3(b) the input width was $\tau=2$, so that the initial intensity was four times higher than in Fig. 3(a). By comparing Fig. 2 (left) and Fig. 3(b), one also can see that for a fixed input time width, switching of the whole output pulse from the cross to the bar state may be achieved by increasing the input area (or intensity).

The mechanism behind the resistance of SIT solitons to linear coupling is the coherent exchange of energy between the field and the atoms in the input guide, which effectively hides the pulse from the other guide. In a linear directional coupler, energy transfer may be inhibited by introducing strong differential gain or absorption between the input guide and the crossed guide. Here the input guide is transparent because the leading part of a $2 \pi$ pulse in the input waveguide is absorbed, whereas the trailing section of the pulse is amplified by stimulated emission. On the other hand, whenever the coherent pulse reshaping is strong relative to the linear coupling, all the energy that gets coupled to the cross guide is rapidly absorbed. As a result, the growth of energy in the cross guide is inhibited, and the input soliton propagates unchanged. The relative strength between nonlinearity and coupling may be varied by changing the input pulse width, which in coherent pulse propagation leads to different values of the group velocity in the medium. The separation between coupled and decoupled soliton regimes occurs at the critical pulse width

$$
z_{c} \alpha \tau_{p}=1 \text {, }
$$

where $z_{c}$ is the width of the coupling region and $\tau_{p}$ is the critical input width of the $2 \pi$ soliton. In fact, in the retarded coordinate frame of Eqs. (3), the velocity of the soliton is $\alpha \tau_{p}^{2}$. Therefore a time delay of one pulse width occurs over the distance $z_{c}$ of Eq. (6). This is confirmed by the numerical results: Eq. (6) predicts, in real units, $z_{c}=3600 \lambda$ with $\alpha$ and $\tau=$ $\tau_{p}=2$ as in Fig. 2 (left). In conclusion, pulses that are longer (shorter) than $\tau_{p}$ remain uncoupled (cou- pled) through the TLS NLDC. In fact, Eqs. (3) and (4) show that an increase in the input pulse width $\tau_{p}$ is equivalent to an increase in the strength of the nonlinear coefficient $\alpha$.

The crucial advantage of using coherent effects for all-optical processing is the possibility of exploiting the resonant enhancement of the nonlinearity, which would permit relatively low-power operation of the NLDC at an arbitrarily high bit rate. As discussed by Watanabe et al. ${ }^{10}$ bound excitons in CdS may be modeled as a TLS with transverse decay times of the order of hundreds of picoseconds. With an exciton concentration $N=10^{19} \mathrm{~m}^{-3}$, an input $2 \pi$ pulse width of $2 \mathrm{ps}$, and a linear coupling distance of $2 \mathrm{~mm}$, we predict switching energies as low as $0.1 \mathrm{pJ}$. We have also verified that the SIT switching mechanism persists whenever moderate background losses are present (i.e., for $\tilde{\sigma} z_{c} \leq 0.2-0.3$ ).

This research was carried out in the framework of contract SC1-0325-C of the Commission of the European Communities on Space-Time Complexity in Nonlinear Optics and under the agreement between Fondazione Ugo Bordoni and the Istituto Superiore Poste e Telecomunicazioni, with partial support from the Italian National Research Council.

\section{References}

1. S.M. Jensen, IEEE J. Quantum Electron. QE-18, 1580 (1982).

2. S. Trillo, S. Wabnitz, E.M. Wright, and G.I. Stegeman, Opt. Lett. 13, 672 (1988).

3. A. Guzman, M. Romagnoli, and S. Wabnitz, Appl. Phys. Lett. 56, 614 (1990).

4. S. L. McCall and E.L. Hahn, Phys. Rev. 183, 457 (1969).

5. U. Langbein, F. Lederer, and H. E. Ponath, Opt. Quantum Electron. 16, 251 (1984).

6. A.I. Maimistov and E.A. Manykin, Sov. Phys. JETP 58, 685 (1983).

7. F.P. Mattar and M.C. Newstein, IEEE J. Quantum Electron. QE-13, 507 (1977).

8. M. J. Ablowitz and Y. Kodama, Phys. Lett. A 70, 83 (1979).

9. J.A. Fleck, Phys. Rev. B 1, 84 (1970).

10. K. Watanabe, H. Nakano, A. Honold, and Y. Yamamoto, Phys. Rev. Lett. 62, 2257 (1989). 